\title{
El Segundo Cerebro del ser humano
}

The Second Human Brain

Autor

Dr. Holguer Romero Urréa

MASTER EN SALUD MENTAL Y CLINICA SOCIAL

Escuela de Ciencias Sociales y de la Salud

Universidad Estatal Península de Santa Elena (UPSE)

Campus La Libertad, vía principal Santa Elena - La Libertad

La Libertad-Ecuador

Email: rholguer2006@hotmail.com

(1) Dra. Rocío Ponce Delgado MSc

(2) Ps.Cl. Johanna Arellano Romero MSc

(3) Rubén Parra Leones.

\section{Resumen}

Existen dos tipos de cerebros, el conocido por todos formado por el sistema nervioso central, que sería el cerebro donde las emociones se forman en base a las experiencias anteriores. El segundo cerebro, el otro, ignorado por la mayoría de seres humanos es el cerebro que está en los intestinos, Sistema Nervioso Enteral o mesentérico, que se caracteriza por la relación del cerebro y aparato digestivo, este cerebro posee más neuronas que su par, guarda emociones, determina la respuesta de acuerdo al contexto y la intensidad del estimulo.

Este articulo tiene por objetivo principal analizar la participación del segundo cerebro en la conducta del hombre, con la ayuda de una nueva especialidad de medicina, la Neurogastroenterología, con su explicación se determina la reacción que existe entre el cerebro mesenterio y el cerebro central, se identifica a cada uno en sus funciones, se efectúa un análisis referencial de como el estrés influye en la conducta, produciendo una crisis. Que se manifiestan con trastornos fisiológicos y psicológicos, como diarreas o estreñimiento, con dolor y trastornos de ansiedad, cansancio, insomnio, falta de concentración perdida de la memoria y a su vez ulceras gástricas, hipertensión, gases, que no necesariamente se debe solamente a un problema estomacal producido por una mala ingesta de alimentos, sino por comportamientos emocionales que repercuten negativamente en esta parte del cuerpo. Se comprobará la influencia del segundo cerebro en la impulsividady sus daños colaterales en el organismo.

Palabras Claves: Mesentérico, enteral, cerebro, sistema nervioso, ansiedad.

\begin{abstract}
There are two types of brains, made known to all by the central nervous system, which would be the brain where emotions are formed based on past experience. The second brain, the other, ignored by most humans is the brain that is in the bowel, or mesenteric Enteral Nervous System, characterized by the ratio of the brain and digestive system, the brain has more neurons than his counterpart, stored emotions, determines the response according to the context and intensity of the stimulus.

This article aims to analyze the participation of the main second brain in human behavior, with the help of a new specialty of medicine, Neurogastroenterology, with its explanation determines the reaction between the brain and the central brain mesentery is identifies each one in its functions, is analyzed as referential stress affects behavior, producing a crisis. Manifested with physiological and psychological disorders, such as diarrhea or constipation, pain and anxiety disorders, fatigue, insomnia, poor concentration and memory loss turn gastric ulcers, hypertension, gases, not necessarily due only to a stomach problem caused by poor food intake, but by emotional behaviors that adversely affect this part of the body. A test for the influence of the second brain in impulsivity and its collateral damage in the body.
\end{abstract}

Keywords: mesenteric, enteral, brain, nervous system, anxiety. 


\section{Introducción}

Entender el funcionamiento del cerebro, en su complejo mundo del sistema nervioso sus partes integrantes y constitutivas, produce un grado de dificultad para quienes no tienen una base de conocimiento en biología y anatomía en su preparación académica, saber sobre el bulbo raquídeo, protuberancia anular, medula espinal, se nos hace difícil estructurar en nuestra mente, como participa este complejo sistema en coordinación con otros sistemas como el endocrino, el inmunológico en la conducta del ser humano, el proceso estimulo respuesta es el resultado de redes de información que se intercambian de manera precisa y exacta, en nuestro cerebro se da la producción de respuestas que el organismo adopta de manera automática; esto hemos aprendido toda la vida, pero, ahora se ha determinado que el ser humano no solo tiene un cerebro, varios científicos lo confirman que tiene dos cerebros, aceptarlo resulta irrisorio, pero lo explica cuando se pregunta el porqué de la conducta incierta, existen respuestas que no están acorde con el aprendizaje, con el estilo o forma de actuar, respuestas ante los porqué actué de esa manera si no es mi forma de ser? " "eso yo no quería decir", "no quise hacer eso", "es que tenia tanto coraje que las palabras me salieron solitas", "no pude aguantar después de haber escuchado tantas tonterías, que lo insulte y le impedí que siga". Se ha escuchado en muchas relaciones sociales de varios contextos terminologías como, "eres una persona instintiva", "eres muy visceral".

Esas palabras escuchadas serán nuestra explicación para determinar la existencia del segundo cerebro, y la participación que tienen nuestras viseras en la conducta humana instintiva. Es el propósito describir el complejo sistema cognitivo; que como el ser humano reacciona ante los diferentes estímulos que existen en el medio externo o contexto, está representado por el ecosistema, formado por el micro sistema o contexto familiar propio, meso sistema formado generalmente por el contexto familiar de origen, el exo sistema llamado así porque la influencia en la conducta en el individuo es menor, y el macro sistema, que están representadas por las leyes y las diferentes normas a las que estamos sometidos como miembros de una sociedad o país.

Se efectuara un estudio descriptivo y comparativo de la participación del cerebro central o primer cerebro y el segundo cerebro o entérico, en el proceso interactivo con todos los estímulos, sean estos internos y/o externos, en la selección de

Respuestas, conoceremos el funcionamiento de cada uno de ellos y entre sí, e independientemente, entre la conciencia y el contexto para concluir en la determinación del tipo de conducta
Ante este tipo de sistemas se ha desarrollado como seres humanos respuestas aprendidas a través de un proceso de aprendizaje consciente e inconsciente que esta acumulado en el hipocampo, para ser utilizado en un momento especifico.

Este es el conocimiento general, pero en este trabajo de investigación se comprobará que no solo se guardan las emociones y el conocimiento en el cerebro superior, sino también se guarda esta información en la viseras, en el sistema nervioso inferior, en el cerebro bajo; mientras más conocimientos o experiencias guardemos a nivel del segundo cerebro mas difícil resultara controlar la impulsividad.

La probabilidad de ser impulsivos se incrementa cuando nuestras respuestas son rápidas, sin razonamiento consiente, mientras el aprendizaje sea menos razonado, más imprevisibles serán las respuestas, las experiencia asociadas con aportes afectivos, producen que los resultados de estas percepciones serán emociones violentas, de tipo físico o fisiológicas.

Conociendo la causa del trastorno de conducta se puede determinar si la acción o respuesta conductual, es normal o anormal, si somos viscerales o somos racionales.

Las fuente bibliográficas que se emplearon en esta investigación, han sido determinadas por los estudios realizados por diferentes especialistas en Neurología, psiquiatría, psicología y gastroenterología, en algunos países existe especialidades como psiquiatría gastroenterológica, que nos brindara una amplia visión sobre la problemática planteada, la amplia importancia del tema ha permitido el avance de las neurociencias en el tratamiento etiopatogénico con resultados duraderos.

Cuando existe el predominio del cerebro mesentérico en la conducta del individuo, existe la prevalencia de la impulsividad, esta producen alteraciones en el organismo como disfunciones o enfermedades de tipo crónico, con la finalidad de confirmar este concepto se aplica la Escala de Control de los Impulsos Ramón y Cajal a pacientes con diferentes patologías de tipo crónico en especial en disfunciones gastrointestinales, para determinar la escala de impulsividad y control de las emociones que darán como resultado un comportamiento especifico.

En la actualidad se conoce que la mayor cantidad de células nerviosas, de neuronas, que posee el cuerpo humano, están ubicadas en el segundo cerebro y en este sector también se acumulan emociones y una cantidad específica de información que intervienen en la conducta del ser humano. 


\section{El Cerebro Mesentérico}

Para iniciar la descripción, y función del segundo cerebro es necesario describir de una manera sistematizada, esquematizada, el mecanismo del sistema nervioso central, este macro sistema nervioso es necesario reconocerlo como principal y centro de todas las funciones psíquicas con predominio de las funciones intelectivas, el reconocimiento básico será descrito con el fin de especificar las funciones biológicas, o fisiologías de nuestros organismo en relación con los diferentes órganos, aparatos y sistemas incluyendo al sistema digestivo. Partiendo de un principio mecanicista debemos entender que en si solo el sistema nervioso central, SNC, determina la conducta del ser humano, estas redes de información son intercambiadas con la naturaleza, con el exterior, estos dos procesos donde intervienen las células nerviosas, cada uno actuando de una red cerrada entre ellos, son modificados o interpretados en el interior por el grado afectivo que le damos a ese estimulo.

A pesar de su imagen estrictamente mecanicista, Ross Ashby adelantaba la incipiente disciplina de la ciencia cognitiva con su detallado análisis de sofisticados modelos cibernéticos de procesos neuronales. En particular, admitía claramente que los sistemas vivos son energéticamente abiertos y al mismo tiempo, en terminología actual, organizativamente cerrados: en los estudios de Ross se compara el sistema vital como una máquina de precisión.

«La cibernética podría... definirse», escribía Ashby, «como el estudio de sistemas que están abiertos a la energía pero cerrados a la información y al control, sistemas que son "estancos a la información".»1

El aporte de Ross Ashby explica que cada sistema mantiene una tipo de información, independiente, el contexto externo o la naturaleza posee el objeto, que no es ni malo ni bueno en ese sector, red cerrada de información, al ser captado por los órganos de los sentidos ese objeto es percibido, ingresa en un macro sistema de energía abierta formado por una red de neuronas, ellas guardan la información, pero en su ingreso al cerebro el sector de la amígdala cerebral provee de una carga afectiva a esta información adquirida, que proviene de otro circuito cerrado de información, al relacionarse las dos, la influencia afectiva consigue que la información ingresada por la neuronas adquiere un concepto de bueno o de malo.

\footnotetext{
${ }^{1}$ CAPRA F., (1998)
}

La conducta no está dada solo por los sistemas independientes, está formado por la relación con el contexto, tanto social como interno.

\section{Sistema neurológico.}

El tipo de sistema neurológico en su estructura interna, se clasifica como fuerte o débil y determina el tipo de respuesta del organismo al contexto, determinando la conducta y para el conocimiento global se estudiará su estructura, las funciones psíquicas necesitan la parte física para poder existir, es el sistema nervioso, el cerebro como elemento principal, en este tema se estudia las funciones y algunas concepciones sobre el sistema neurológico, tomando en cuenta que las funciones de la corteza cerebral en su totalidad aun no están bien determinadas.

\section{Descripción anatómica.}

"El cerebro central o primer cerebro, está formado por los hemisferios cerebrales, ocupan la mayor parte del interior del cráneo, representan aproximadamente un $80 \%$ del peso del cerebro, contienen cerca de $70 \%$ de la neuronas, la cisura inter-hemisféricas, divide al cerebro en dos, el hemisferio cerebral derecho e izquierdo, estos están unidos entre sí por sus fibras inter-hemisféricas siendo la más importante la que corresponden al cuerpo calloso. Cada hemisferio cerebral esta subdividido en cinco partes por diversas cisuras, los lóbulos que llevan el nombre de los huesos que le dan cabida, lóbulo parietal, lóbulo occipital, lóbulo temporal, lóbulo frontal, ínsula, límbico; el insular y el límbico no son verdaderos lóbulos. El lóbulo límbico es el que se relaciona en particular con funciones viscerales y de la conducta.

Todos los lóbulos cerebrales cumplen una función específica, una es la continuación de la otra, pero debe entenderse que con la ausencia de uno de ellos aun las funciones psíquicas y el mismo ser humano continúan en una relación con el medio o entorno, se han realizado lobotomías completas de un hemisferio y el individuo puede vivir una vida relativamente normal con alteraciones leves en la psicomotricidad y áreas sensoriales, con independencia de la dominancia cerebral.

\section{Lateralización.}

El propósito de este estudio es determinar el tipo de redes de información que se transporta por el sistema nervioso en general, al estudiar la Lateralización cerebral del encéfalo, debo dejar en claro que lateralidad completa se entiende por el común uso del pie, mano, oído y ojo del mimo lado del cuerpo, solo ocurre el ser humano que es capaz de hablar, leer y escribir; una característica propia del encéfalo humano es la distribución de las funciones en los hemisferios cerebrales, a simple vista el encéfalo parece tener una simetría bilateral perfecta, como la mayor parte de los órganos del cuerpo, por ello, cabría esperar que las dos mitades del encéfalo también cumplieran funciones 
equivalente, muchas funciones más especializadas las lleva a cabo un solo hemisferio, simétricamente. ${ }^{2}$

Nuestra mente funciona estableciendo relaciones, contactos, hipervínculos, y mantiene un peculiar equilibrio entre los elementos que relaciona, entre las partes y el todo, entre los hemisferios derecho e izquierdo del cerebro se produce la inevitable interdependencia entre las notas musicales y la música: las partes carecen de sentido sin la totalidad y viceversa.

Los físicos aseguran que lo más íntimo de la materia y del universo está gobernado por la simetría. Sin embargo, los investigadores más cercanos a la biología afirman que la asimetría es lo que realmente nos hace humanos, el cerebro es asimétrico y hasta los besos son asimétricos. Las desigualdades anatómicas son, además discretos portavoces de la biología del individuo, de su personalidad, y determina el poder de atracción para el sexo opuesto. "La forma del cerebro se adapta al uso que le dé su portador, todos los cerebros incluyendo a los cerebros de seres inferiores" 3

El hemisferio cerebral izquierdo no se refiere a la especialización del lenguaje como tal, sino a una especialización en el procesamiento y manejo de ciertos parámetros de la información que habitualmente se haya contenida en el lenguaje, en lo referente a los códigos, sean fonéticos o simbólicos.

Desde hace mucho tiempo se ha considerado que el hemisferio cerebral derecho es el hemisferio menor o no dominante. Esto se debió en su tiempo a que las lesiones que se habían dado en el hemisferio izquierdo (área de Broca) generalmente producen trastornos en el habla o en la lectura y no se observan cuando ocurren iguales traumatismos en el área del hemisferio derecho, pero en otras investigaciones determinaron que las lesiones producidas en el hemisferio cerebral derecho producían alteraciones a nivel emocional e intelectuales. Todas estas alteraciones, las cuales implican cambios y anomalías emocionales, son características del hemisferio cerebral derecho. Sin embargo, es posible señalar un segundo tipo de daño, el cual se caracteriza por modificaciones profundas del estado emocional del paciente. Nos referimos a las lesiones de las regiones pre frontal.

La actividad de los lóbulos frontales se ha relacionado con las siguientes funciones: la regulación del nivel de activación (Homskaya,1972; Pribram,1973; Ardila,1997); la organización de programas motores especialmente cuando se hallan bajo el control del lenguaje ${ }^{4}$ la organización de los aspectos temporales y secuencias de la memoria y el comportamiento (Iversen, 1972), la utilización de información

\footnotetext{
${ }^{2}$ Romero H 2010

3 Romero H. 2013

${ }^{4}$ Luria A, 1963, 1967,1977
}

espacial (Konorski y Lewicka,1964), solución de problemas (luria y Tsvetkova 1967), y el control de los elementos afectivos y emocionales del comportamiento (Luria,1977; Walsh,1978). ${ }^{5}$

El Hemisferio Cerebral Izquierdo procesa estímulos relativamente tenues y en rápido movimiento, en cambio en el Hemisferio Cerebral Derecho procesa estímulos mucho más intensos e inmóviles, inclusive los estímulos claramente estáticos.

$\mathrm{Al}$ exponer un concepto o una palabra ante un grupo indeterminado de individuos sin que exista entre ellos ningún factor común en sus características neuroanatómicas y neurofisiológicas, se observa que el mismo estímulo es captado por cada uno de diferente manera, dependiendo para ello de sus características individuales, de su entorno, de su mismo ADN, el factor hereditario y el cromosoma $\mathrm{x}$ determina la diferencia en la especialización o captación de estímulos, y la respuesta será única e individual.

El tipo de sistema nervioso se forma desde el mismo instante de la concepción, ello confirma la existencia de un arco reflejo perfecto, con órganos receptores, vía aferente y vía eferente, que van especializándose en base a la ejercitación o el uso cotidiano de sus facultades intrínsecas, por medio de los órganos de los sentidos, el ser humano se relaciona con el entrono y se especializa.

Con estas características analizadas se puede comprobar cuál es el hemisferio que más se desarrollan en las diversas tareas presentadas, en eventos o vivencias que están relacionados con procesos sensoriales, preceptúales, cognitivos y con su tiempo de reacción.

El neurólogo Roger Sperry, luego de una etapa de aproximadamente dos siglos de materialismo dialectico o científico se estudia la relación entre la materia o sistema nervioso integral en relación con el medio, creando nuevos principios cognitivos de nosotros mismos, con estos estudios la ley del determinismo y fenomenología, prevalece en el estudio de las ciencias como la gnoseología, psicología y las llamadas neurociencias en el año 1995, en sus investigaciones determina que: "ambos lados del cerebro son diferentes y que tienden a dividirse las principales funciones intelectuales".

Lo que le lleva a Sperry a afirmar que: “... En contra posición a visiones fisicistas anteriores, los nuevos principios de causalidad afirman que los valores subjetivos del ser humano constituyen hoy la fuerza estrategia más poderosa que gobierna los sucesos en el mundo, clave de nuestro predicamento global y de su solución.” 1995.

El estudio de los principios del determinismo nos obliga a buscar la etiología de la patología en la relación básica del interior o función del cerebro con

${ }^{5}$ Luria A, 1977 
el exterior o el contexto por tanto Sperry conceptúa que "El hemisferio derecho es dominante en los aspectos del intelecto, percepción visual y espacial; o la captación total de las imágenes, por lo que se le conoce con el nombre de intuitivo, es el sintetizador, el de la estructura total y la imaginación. Es el hemisferio no verbal, reconoce caras, voces, melodías, piensa y recuerda en imágenes. El hemisferio izquierdo se especializa en funciones verbales y matemáticas, es el analizador, es lógico y secuencial". Es analítico, se encarga de procesar la información. Razones por las que se lo conoce como el hemisferio racional.

Jerry Levy 1995 descubrió que el procesamiento de ambos hemisferios a pesar de sus diferencias es comparable en complejidad. Cada mitad tiene su propia forma de conocimiento y su manera de percibir el entorno.

$\mathrm{Al}$ igual que las funciones de cada hemisferio, hoy nos motiva la participación del cerebro mesentérico en el control de las emociones humanas, con su sistema nervioso formado por las mismas neuronas.

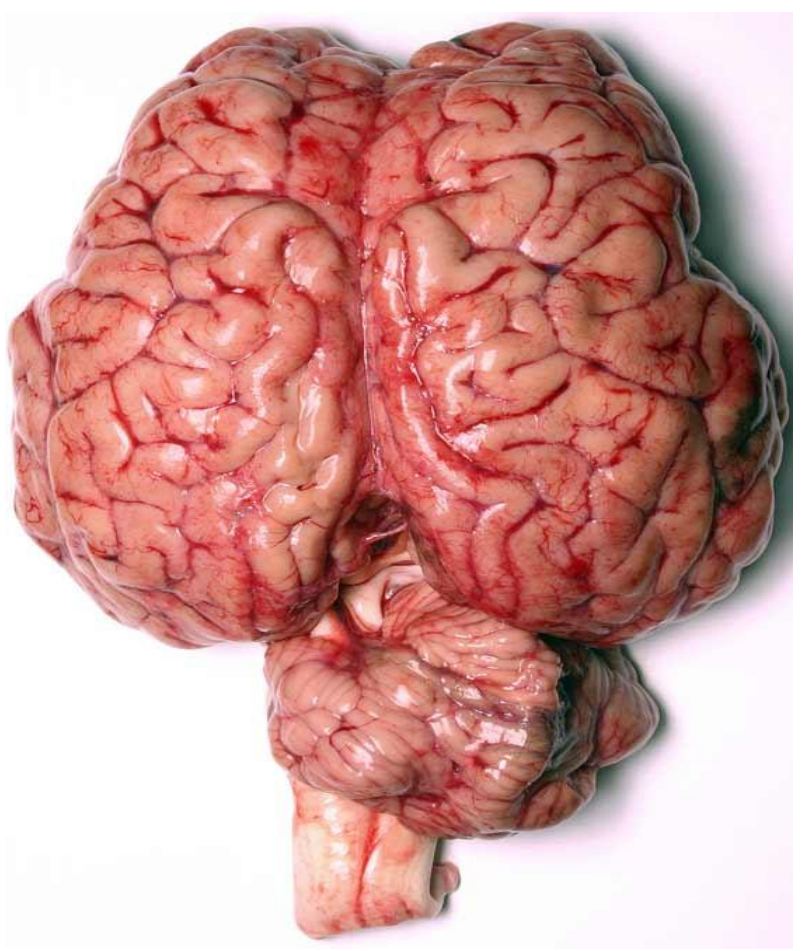

Fig. 1. Fotografía de un cerebro humano extraído en una autopsia a pocas horas del deceso.

Fuente.upload.wikimedia.org./Wikipedia/commons/thumb I

Con todo lo expuesto queda establecido el origen de las funciones psíquicas y la relación que existe entre los diferentes órganos aparatos y sistemas. Este es el conocimiento que hasta hoy ha sido divulgado ahora iniciaremos una aventura por el cerebro mesentérico.

\section{Cerebro Mesentérico.}

Un artículo reciente publicado por la revista Scientific American analiza los conocimientos más recientes sobre esta red neuronal y explica las características del técnicamente conocido como "sistema nervioso entérico". Conocido como el "segundo cerebro", consiste en una red de neuronas, a menudo pasada por alto, que recubre nuestras vísceras. La profundización en el conocimiento de esta masa de tejido neuronal formada por importantes neurotransmisores está revelando que este segundo cerebro hace mucho más que controlar la digestión de alimentos.

Se encuentra situado en nuestros intestinos, y está compuesto por 100 millones de neuronas. La revista Scientific American analiza el curioso funcionamiento de esta red neuronal, gracias a la que "sentimos" ciertas emociones en la tripa, y también gracias a la que nuestro organismo hace la digestión sin contar con la ayuda del cerebro principal. En la revista se analizan asimismo los resultados de las últimas investigaciones al respecto, que señalan que el segundo cerebro estaría relacionado con diversas enfermedades, como el autismo o la osteoporosis. Por Yaiza Martínez.

El estudio del segundo cerebro ha dado lugar, en las últimas dos décadas, a una rama de investigación conocida como neurogastroenterología. La importancia de conocer a fondo el sistema nervioso entérico radicaría en que éste se encarga de que las funciones digestivas mantengan un funcionamiento coordinado e integrado.

Pero no sólo eso, afirman los expertos. Situado en el interior de las entrañas, el segundo cerebro está conectado con el cerebro situado en el interior del cráneo $\mathrm{y}$, al menos en parte, determina nuestro estado mental y juega un papel clave en ciertas enfermedades del organismo.

Fuera de su alcance quedan, a pesar de la gran repercusión que esta red neuronal tiene para el cuerpo, la generación de pensamiento consciente y la capacidad de decisión, afirman los investigadores. En 1999 el profesor Michael Gershon de la Universidad de Columbia, de Nueva York, publicó un ensayo tras haber estudiado durante 30 años el sistema nervioso entérico. Descubrió que tenemos cien millones de neuronas entre dos capas musculares del tubo digestivo, que son totalmente idénticas a las del sistema nervioso central (el cerebro superior). Estas neuronas tienen el mismo lenguaje neuronal que las del cerebro y producen los mismos neurotransmisores (sustancias químicas destinadas a producir algún efecto). Lo más interesante de esto ha sido descubrir que el $90 \%$ de la serotonina (la famosa hormona de la felicidad y el bienestar) se produce y se almacena en el sistema nervioso entérico, o cerebro digestivo. También tenemos ahí sustancias parecidas a las benzodiacepinas, lo que quiere decir que tenemos poder ansiolítico (tranquilizante) en la tripa. Este segundo cerebro tiene un vínculo profundo con 
nuestras emociones y nuestro bienestar. Endorfinas efecto en el bienestar del individuo.

Según declara el experto en la materia Michael Gershon, del Columbia University Medical Center: "el segundo cerebro no ayuda en los procesos mentales: la religión, la filosofía y la poesía están en manos del otro cerebro". Gershon está especializado en el estudio de esta red de neuronas, y es autor de un libro titulado "The Second Brain".

Los intestinos forman parte del segundo cerebro y cumple papeles definidos, el intestino delgado separa lo bueno de lo malo y en él se absorben las sustancias nutritivas. Esto supone un proceso de integración física y también de sentimientos, pensamientos y experiencias. Los síntomas intestinales pueden reflejar la personalidad y los conflictos psíquicos.

La inseguridad, el miedo y otros factores similares producen retención y los consiguientes estreñimientos, úlceras intestinales o colon espástico. En el intestino, donde se conectan las realidades interna y externa, se pueden retener aspectos de la propia personalidad que da miedo liberar. La obsesión por controlar impide la espontaneidad. Los cambios y los viajes, por la sensación de desprotección que conllevan, pueden ir acompañados de molestias intestinales. ${ }^{6}$

\section{Estructura y origen del Sistema Nervioso Entérico.}

Ese sistema nervioso está compuesto por conjuntos de neuronas incrustadas en las paredes del largo tubo del intestino, o canal alimenticio, cuya extensión es de alrededor de nueve metros, desde el esófago al ano. ${ }^{7}$

Este segundo cerebro contiene alrededor de 100 millones de neuronas, que es más de las que hay en la médula espinal $o$ en el sistema nervioso periférico, explica Gershon.

Esta enorme cantidad de neuronas del sistema nervioso entérico es la que permite que la digestión se haga de forma apropiada, sin que el cerebro principal tenga que intervenir en sus procesos: la rotura de los alimentos, la absorción de los nutrientes y la expulsión de los desechos requieren de procesos químicos, contracciones musculares, etc. de los que se encarga el segundo cerebro, de forma independiente.

Pero la complejidad del sistema nervioso entérico no termina ahí, porque este segundo cerebro también influye en una gran parte de nuestras emociones: la sensación de tener "mariposas en el estómago", por ejemplo, es en realidad una señal fisiológica que responde a un estado de nervios o de estrés, y que percibimos gracias los nervios presentes en nuestro intestino.

6 Pag 34 Perception and reflex responses to intestinal distension in humans are modified by simultaneous or previous stimulation 7 J SIERRA P, (2001)El cerebro intestinal.
También hay evidencia de la existencia de comunicación del sistema nervioso entérico con el sistema inmune, en la llamada intercomunicación inmuno neuronal ${ }^{8}$.

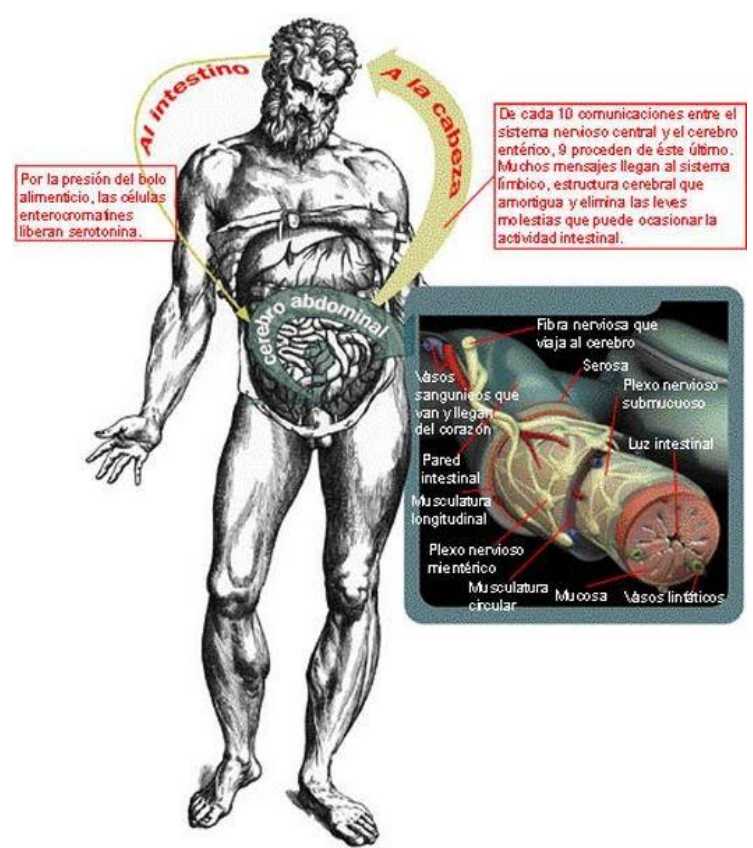

Fig 2. El sistema nervioco central y el sistema nervioso entérico. Fuente: Nuestro segundo cerebro/www.zonade.com

Con respecto a su origen, el doctor Alcázar cuenta que el médico estadounidense Max Gerson (creador de la Terapia de Gerson) planteó estudios que "demostrarían que en el intestino existiría un segundo cerebro" y para explicarlo mejor, dice que este especialista mencionó haber descubierto que en esta parte del cuerpo, "se produce el $90 \%$ de la serotonina, que pensábamos era un neurotransmisor solamente del cerebro".

En nuestra sociedad, el ser humano recibe un exceso de estímulos que percibe día a día, intercambian información, produciendo con el contexto en el que se desarrolla generando un aprendizaje con relación de afecto con el objeto de análisis, o con el estimulo percibido, la estimulación produce una respuesta positiva o negativa dependiendo del bagaje cognitivo que hemos acumulado durante toda nuestra vida este conocimiento influye en la respuesta y este a su vez influye en el proceso del sistema nerviosos mesentérico o cerebro intestinal y en el equilibrio inmunitario que este realiza, poniéndose al descubierto a una serie de microrganismos hostiles, esta es una de las causas para que las personas se

${ }^{8}$ J SIERRA P, (2001) 
enfermen de gastritis, alergias, gripes, etc. es por eso que la percepción está determinada por el pre concepto, no hay nada malo en el contexto externo, ni nada malo, todo fuera de nuestra conciencia es perfecto, somos cada uno de nosotros quienes cambiamos el sentido del concepto del estimulo, vemos lo que queremos ver. Nos enfermamos porque queremos que el mundo externo sea como nosotros deseamos, queremos cambiar en nuestra mente el plomo en oro.

La complejidad del segundo cerebro hace que, según Gershon, de él dependa en parte nuestro bienestar físico y emocional cotidiano.

Dada la concordancia entre ambos cerebros, los científicos afirman que, por ejemplo, ciertos tratamientos contra la depresión que tengan como objetivo la mente, pueden impactar sin pretenderlo en el intestino.

Es el caso de ciertos medicamentos antidepresivos, los denominados inhibidores selectivos de la receptación de la serotonina (ISRS), que aumentan los niveles de serotonina en el organismo, y que pueden provocar efectos gastrointestinales secundarios, como el síndrome del intestino irritable.SII ${ }^{9}$

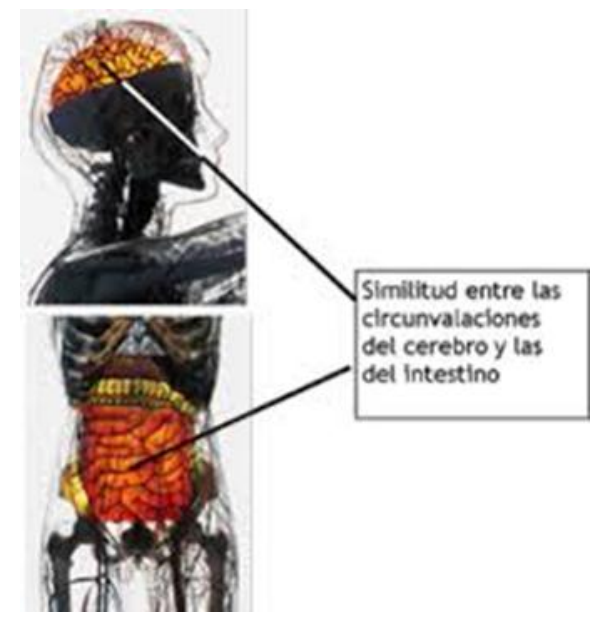

Fig. 3. Comparación entre los dos cerebros en su extructura. Fuente: Seres libres.com

Esto es debido a que la serotonina, una hormona que es sintetizada por el sistema nervioso central y que actúa como neurotransmisor, se encuentra en su mayor parte (en un 95\%) en el tracto intestinal del organismo.

Los científicos están descubriendo que la serotonina podría, además, jugar un papel en la aparición de otras enfermedades. Recientemente, en la revista "Nature" apareció un artículo que relacionaba un medicamento inhibidor de la producción de serotonina en el intestino con la osteoporosis (enfermedad ósea degenerativa) en roedoras postmenopáusicas.

\footnotetext{
${ }^{9}$ SIERRA P, (2001)
}

Asimismo, la serotonina del "segundo cerebro" podría jugar un papel en el autismo. En sus investigaciones, Gershon ha descubierto que los mismos genes implicados en la formación de la sinapsis (conexiones entre neuronas) del cerebro están también implicados en la formación de sinapsis del sistema nervioso entérico. Según él, si estos genes se ven afectados por el autismo, esto podría explicar por qué muchos niños autistas padecen anomalías gastrointestinales.

Otras investigaciones actuales tratan de establecer cómo el segundo cerebro interviene en la respuesta inmune del organismo, dado que el $70 \%$ de nuestro sistema inmunológico se centra en el intestino para expulsar y matar a sus invasores, un trastorno a este nivel podría causar un desequilibrio homeostático y podría afectar a todos los órganos aparatos y sistemas.

La Dra. Irina Matveikova (autora del libro "Inteligencia Digestiva") afirma que en general las personas con Síndrome de Colon Irritable, SCI, suelen ser hiperactivas, nerviosas, con tendencia a las fobias y con problemas de autoestima, pero aún no se conoce cómo tiene lugar el proceso: si es la personalidad lo que provoca el trastorno o si es el trastorno el que condiciona la personalidad.

$\mathrm{Si}$ aplicamos lo expuesto en el sistema nervioso central, el principio o ley universal del determinismo y la fenomenología siempre existirá una interacción entre los sistemas nerviosos y la conducta será modificada por la información guardada en el cerebro mesentérico, rencores, resentimientos, ira, agresividad de todo tipo.

\section{El estrés y los procesos digestivos.}

Con el mecanismo de estimulo respuesta y con las leyes de causa efecto queda determinado que las alteraciones físicas son el resultado del estrés, la mayoría de médicos clínicos muchas veces sólo se centran en los malestares fisiológicos que produce un contexto saturado de estímulos intensos, se preocupan de aliviar síntomas, pero no la causa que lo produce, ya sea esto por los problemas pasados o los problemas que esté viviendo en el presente por la dificultad que tienen para resolver sus problemas o tomar decisiones.

Una idea fija por un problema no resuelto produce un desgaste permanente del sistema inmunológico y de energía traducida en vitaminas, proteínas y minerales que agravan las respuestas de tensión, fatiga y dolor muscular u orgánico.

En relación de la mente y el cuerpo se puede citar un ejemplo como es el caso de la gastritis y el proceso etiopatogénico se lo puede explicar; Cuando un individuo se muestra ansioso, excesivamente preocupado, con una idea fija, obsesiva, puede provocar una alteración en las células que se encuentran ubicadas en la zona muscular y llegar por medio del sistema linfático hasta el centro mesentérico, bajan hacia el estómago produciendo como respuesta la producción de jugo gástrico 
similar a la respuesta que se produce cuando espera el bolo alimenticio, al no llegar el bolo, el acido que iba a desintegrarlo, queda en el fondo del estomago y empieza a quemar su superficie, se intensificara cuando por ansiedad trague gran cantidad de saliva. El estrés influye en los procesos normales de la digestión ocasionando una lentitud en las respuestas motoras, en la rapidez de razonamiento, en la agilidad mental en general, el ser humano puede estar ingiriendo los alimentos bajo presión emocional o circunstancial, la lentitud confirma que las encimas hepáticas tienen complicaciones en los procesos de transformación de los alimentos. Sumando a esta sintomatología. Cuando existen desórdenes alimenticios la patología por estrés se incrementa, porque sin alimento la capacidad de rendimiento del individuo disminuye.

Al hablar de estrés y los efectos que este acarrea sobre el organismo, se nos viene a la mente un sinnúmero de manifestaciones tanto fisiológicas como psicológicas que son producto de su acción, dentro de los que podemos encontrar: fatiga, dificultades de las relaciones interpersonales, baja del sistema inmunológico, algias y mialgias, cefaleas, temor con rasgos paranoides, hipertensión arterial, hipersensibilidad, erupciones cutáneas, trastornos de sueño, déficit de atención, ideas fijas de invalides e incapacidad, inseguridad, principal causante de muertes por cáncer, enfermedades cardiacas, cirrosis hepática, enfermedades pulmonares, eventos cerebro vasculares, accidentes de alto riesgo, sea laboral familiar o de transito por la reunión de signos y síntomas anteriores, suicidios.

\section{Explicación etiopatogenica.}

Con el análisis clínico y fisiológico, hemos determinado signos y síntomas que nos describe una tipo de personalidad específica que pueden ser afectados con mayor intensidad en el área del sistema nervioso mesentérico, las personas tienen un sistema nervioso central débil, con escasa capacidad para enfrentar el estrés, siendo esta característica un factor predisponente, coinciden además con una conducta de ansiedad o angustia, ideas fijas y obsesivas, que como hemos analizado afectan al sistema nervios entérico, con esta sencilla explicación etiopatogenica, hemos iniciado un estudio de varios pacientes gástricos, que acuden a consulta de diversos hospitales.

\section{Resultados}

La investigación realizada esta basada exclusivamente en la observación y encuestas a pacientes que refieren daños a nivel gastrointestinal, para determinar el factor común entre la impulsividad y el daño orgánico y confirmar el control de las emociones en el sistema entérico, se ha utilizado un test de "Control de Impulsividad de Ramón y Cajal".

Muestra. hemos tomado como muestra 18 pacientes de consulta externa de la especialidad gastroenterología de un hospital de la ciudad de
Guayaquil, 18 pacientes con alteraciones gástricas de la ciudad de Libertad y Santa Elena, 18 personas determinadas en la población de Libertad y Santa Elena, al azar.

Se tomo esta muestra de pacientes con esta característica. 18 pacientes profesionales, entre los cuales constan, docentes, sastres, jubilados, militares incluidos entre ellos marinos, policías y de la Fae, quehaceres domésticos, comerciantes abogados estudiantes.

Otra variable analizada en este grupo de trabajo será la edad, la hemos tomado en grupos etarios de 20-35 años, 35-45 años, 45-55 años, 55-65 años y de 65 años en adelante.

Metodología. El primer paso ha sido determinar la relación entre el sistema nervioso central y el sistema nervioso mesentérico, se establece la participación de cada uno de los órganos, aparatos y sistemas en la conducta,

Antes de aplicar el test se entrevista al paciente, se investiga sobre su estado de salud, interesándose más por enfermedades relacionadas al sistema gastrointestinal, ¿si tiene o no afecciones a sus intestinos, el tipo molestias en el estomago o en los intestinos, en general a nivel digestivo.

Hemos tenido la participación de estudiantes de psicología quienes participaron en la recolección de datos del proceso.

Hemos obtenido como resultado que:

a. En este tipo de pacientes con afecciones gastrointestinales existe un predominio de falta de control de la impulsividad,

b. Las personas reaccionan afectivamente con tensión,

c.. No resuelven sus problemas y lo mantiene en un estado de rumiación con su dolor hasta cuando es exteriorizado.

d. Las respuestas emitidas son impredecibles, total mente equívoca, impulsiva, ante un estimulo que no tiene la una alta intensidad, ni guarda relación con el episodio que está viviendo.

e. Los profesionales que mayor impulsividad manifiestan son los militares y los policías, con un $27,7 \%$.

f. Los estudiantes presentan un grado de impulsividad de $16.6 \%$.

g. El menor índice de impulsividad se obtiene en quienes se dedican a los quehaceres domésticos que corresponde al $5.5 \%$, al igual que los jubilados y los abogados en el orden presentado.

h. Las enfermedades que presentan los pacientes con un alto índice de tensión e impulsividad son: con gastritis $42 \%$, con diabetes $32 \%$, con hipertensión $15 \%$, con retención de líquidos 7 , con alergias 3\%, con miopía $1 \%$.

i. En mayor índice de impulsividad se presenta en pacientes con diabetes con un $31.25 \%$, seguido de pacientes con gastritis $25 \%$, hipertensión $16 \%$, los encuestados que presentan diabetes o 
miopía presentan un grado mínimo de impulsividad que corresponde a un $6.25 \%$.

j. Como resultado del análisis etario se obtiene un grado de impulsividad mayor en el grupo de 2035 años con un $44.4 \%$, a esta edad el ser humano tiene energía vital y están dispuestos a obtener sus metas utilizando todos los medios, es el tiempo en el cual todos buscan la culminación de su carrera y de su hogar, a escogido su profesión y un compañero-a; seguidos en el grado de intensidad tenemos a los jovenes de $35-45$ años con un $38,8 \%$, se observa un control de impulsividad en la edad de inicio de la madures, es tiempo de disfrutar el resultado de su trabajo en la juventud, disfrutar del crecimiento de sus hijos, de 45 a 55 años de edad, se intensifica el control de impulsividad a un $11.11 \%$. los hijos están grandes, profesionales y algunos les dieron nietos, es tiempo de darse tiempo para ellos, una gran mayoría de ellos se divorcian para disfrutar una segunda vida, con una vida relajada, emocional y económicamente solvente.

\section{Conclusiones}

a. De lo referenciado en la investigación bibliográfica se ha confirmado. que el cerebro central y el mesentérico participan en las respuestas emocionales y coadyuvan en la elaboración de la respuestas del sentimiento,

b. Con análisis efectuado se ha comprobado que existen la misma estructura nerviosa en los dos cerebros inclusive, existen más neuronas en el cerebro mesentérico,

c. Se ha comprobado la participación de cada cerebro en su responsabilidad de emitir respuestas adecuadas a la intensidad y la frecuencia de sus estímulos y al grado afectivo que resulta de esta relación.

d. Se confirma que todos los cerebros, llamados así el hemisferio cerebral izquierdo, derecho, al cerebro mesentérico, intervienen en una red de información, existe prevalencia de uno de los cerebros en respuestas específicas dependiendo al grado de intensidad del factor emocional, mientras más alta es la escala emocional menor es la participación intelectual, es más instintiva.

e. En la práctica de investigación se confirma que existen profesiones como las fuerzas armadas y policía, que por su tipo de trabajo presentan un mayor grado de ansiedad e impulsividad, que se traducen en la manifestación de trastornos fisiológicos como desencadenantes en esta profesión la impulsividad desarrolla, colon nervioso, gastritis, ulcera.

f. Existe relación en las personas que presentan mayor ansiedad e impulsividad, con las enfermedades de origen psicosomático, estas personas por lo general son de personalidad histriónica, es un persona muy hábil para crear invenciones en base de un acto sencillo de resolver, buscando llamar la atención, tienen la particularidad de sobredimensionar los estímulos percibidos por ende la respuesta en el organismos será más lesiva.

g. La neurofenomenología de Varela plantea que las sensaciones que el ser humano experimenta en la vida cotidiana no activan una determinada zona $x$ en el cerebro como región única, que se corresponda con el tipo de sensación que se experimenta, sino que, al momento de tener una experiencia determinada, distintas zonas del cerebro son estimuladas formando un patrón único que se corresponde con la experiencia irrepetible. $^{10}$

El doctor Alcázar menciona que el síndrome del colon irritable se presenta en un mayor número de mujeres que de hombres ( la relación sería de 3 a 1) y afectaría más a quienes se muestran perfeccionistas, aprehensivos, nerviosos, detallistas y con una necesidad de controlar las cosas. ${ }^{11}$

h. En las enfermedades encontradas en los pacientes con impulsividad, se encuentra no solo una enfermedad en algunos pacientes, se presenta diabetes, hipertensión, retención de líquidos y miopía, una enfermedad conduce a otra, los pacientes con gastritis, presentan retención de líquidos, hipertensión.

i. En los resultados obtenidos en la investigación se encuentra que el índice más bajo de impulsividad y falta de control se presenta en los pacientes diabéticos, esto se debe a la vida sedentaria de estos pacientes, ellos son personas demasiado conformistas, sin retos ni objetivos en su vida, han sido personas sedentarias, oficinistas, en su vida no ha tenido retos, el tipo de respuesta en los reactivos psicológicos se localizan en el tal vez o en alguna vez, reaccionan con impulsividad cuando son afectados en sus necesidades básicas de sobrevivencia, cuando intervienen con su prole, con su derecho a trabajar y a su territorio.

j. En las edades encontramos que el joven que inicia una actividad profesional, familiar, empresarial, en su proceso por adaptarse establece una serie de nuevos parámetros y su adaptación es disminuida, como respuesta a este nuevo medio el sujeto responderá sin mucho razonamiento por el ímpetu y la necesidad de ver resueltos sus conflictos a la velocidad que su edad avanza. A los 35 años a 45 años generalmente el profesional o ama de casa tiene una determinada estabilidad y se encuentra defendiendo su posición, ha disminuido el frenesí, la capacidad intelectiva está en crecimiento y predomina; a los 45 años la madurez emocional y la estabilidad es

\footnotetext{
${ }^{10}$ MATURANA H y VARELA

${ }^{11}$ PAG. 22 El cerebro intestinal
} 
manifiesta en los seres humanos, a esta edad la profesión, la familia y como resultado la capacidad económica estable proporciona una paz y relajación en todos los integrantes de la sociedad de origen y su familia propia, este Aparente equilibrio se comprueba con la disminución porcentual de impulsividad hasta desaparecer por un tiempo; A los 55 años a los 60 se incrementan las patologías psicosociales, porque busca identificarse con un mundo cambiante, quiere ser joven otra vez, para ser aceptado, el hecho de envejecer produce en su vida una conducta de negación, el incremento del temor a morir y no terminar su legado le obliga a desarrollar una agresividad sin control, dirigido a sí mismos y a los demás llegando a ser física o psicológica, la impulsividad se incremente en un $15 \%$ de los adultos mayores, , a esta edad son más propensos los divorcios por incompatibilidad de caracteres, en su mayoría buscan el bien morir, se sujetan de la religión y solo esperan.

\section{Agradecimientos}

Agradecemos la participación de los estudiantes de la carrera psicología de la Universidad Estatal península de Santa Elena, a los y las pacientes y demás personas que participaron en la investigación realizada.

\section{Referencias}

[1] LURIA A. (1999), Introducción: La continua influencia de Alexander Luria en todo el mundo Neuropsicología, Vol 9 No. Marzo 1999 pp 1-7

[2] CAPRA F., (1998), La trama de la vida. Una perspectiva de los sistemas vivos, Editorial Anagrama S.A. Barcelona.

[3] THOMPSON W. (1992), "Las implicaciones culturales de la nueva biología" en Lovelock, J. $y$ otros. Gaia: Implicaciones de la nueva biología. $2^{\circ}$ ed., Editorial Kairós, Barcelona.

[4] FOUCAULT M. (1980), Microfisica del poder. $2^{\circ}$ Ed, Las Ediciones de la Piqueta, Madrid.

[5] IDEM (2008), Filosofía, Ciencia y Saber Andino: Bases ontológicas, gnoseológicas y epistemológicas de la inter e intraculturalidad, $2^{\circ}$ ed. Editorial Universidad Bolivariana, Santiago.

[6] DR. GERSHON, 2007 trasplante del sistema nervioso entérico: Un paso más hacia el tratamiento para aganglionosis. Gut. Apr; 56 (4) :459-61. Revisar.

[7] MATURANA H y VARELA F. (1995), El árbol del conocimiento: Las bases biológicas del entendimiento humano. $12^{\circ} \mathrm{Ed}$, Editorial Universitaria, Santiago

[8] J SIERRA P, (2001)El cerebro intestinal. Servicio de Digestivo. Hospital General Universitario Vall d'Hebron. Barcelona.

[9] Universidad de Buenos Aires facultad de Psicología. Publicada 06/11/2010
[10] MARTÍ E., 2005Segundo Cerebro: Neurogastroenterología. Presidenta Asociación AACICAT Associació d'Afectats Còlon Irritable de Catalunya

[11] SERRA J, AZPIROZ F, MALAGELADA J-R. Perception and reflex responses to intestinal distension in humans are modified by simultaneous or previous stimulation. Gastroenterology 1995; 109: 1742-1749

[12] REVISTA CIENTÍFICA , (2010)Todo Salud Edita: Instituto Biológico De La Salud Médico experto: Dr. Domingo Pérez león, Boletín $n^{\circ} 6 \cdot$ Edición Marzo

[13]ROMERO H., 2010. Los Hemisferios Cerebrales y su dominio, 4 ed. Revista Ciencia de la UNEMI, Universidad Estatal de Milagro. Ecuador. 